were quaternary referrals following failed PEG insertion. Length of stay was $5 \pm 5$ days. Body mass index (BMI) at diagnosis was $27 \pm 5 \mathrm{~kg} / \mathrm{m} 2$ with BMI at PEG insertion $22 \pm 4 \mathrm{~kg} /$ $\mathrm{m} 2$. Mean weight change from diagnosis to insertion was -16 $\pm 10 \%$. Ventilator compliance time was $3 \pm 6$ hours. Eighteen patients were compliant to NIV (NIV usage $>4 \mathrm{~h}$ daily). Thirty-day mortality was $10 \%$ and 90 -day mortality was $15 \%$. Survival time since MND diagnosis for non-complaint to NIV patients $(859 \pm 123$ days) trended to be less than those compliant to NIV $(1165 \pm 203)(\mathrm{p}=0.058)$ (Figure 1$)$.

PEG insertion in MND patients requiring NIV is safe and effective. NIV compliance in this cohort of patients who have bulbar dysfunction might affect survival.

\section{PWE-018 DERIVATION AND VALIDATION OF A ROYAL FREE HOSPITAL GASTROSTOMY RISK SCORE}

${ }^{1}$ Anastasia Russell-Dalton*, ${ }^{2}$ Catherine Fennessy, ${ }^{1}$ Marsha Y Morgan. ${ }^{1}$ UCL Institute for Liver and Digestive Health, Royal Free Campus, University College London, London, UK; ${ }^{2}$ Royal Free Hospital, Royal Free London NHS Foundation Trust, London, UK

\subsection{6/gutjnl-2019-BSGAbstracts.349}

Introduction Percutaneous gastrostomy feeding tubes are used to provide long term artificial nutrition in patients with adequate intestinal absorptive capacity. Placement is associated with low immediate morbidity; however, the early mortality rate is high. Current selection for gastrostomy placement has a significant subjective element. In consequence there is a need for a validated, objective assessment tool, based on easily accessible information, to identify the risk-benefit of gastrostomy placement on an individual basis. The aim of this study was to derive and validate a Royal Free Hospital-Gastrostomy Risk Score (RFH-GRS).

Methods Extensive demographic and clinical data were collected on the 234 patients referred for gastrostomy placement, at the Royal Free Hospital, London, between 1 January 2016 and 31 December 2017; the entire cohort was followed until death or 31 December 2017. Multivariate analysis identified age, multimorbidity (assessed using the Charlson Comorbidity Index), prognosis, and recent infection as independent predictors of survival. Four further factors were identified by experienced nutritional practitioners for inclusion in the RFH-GRS viz. nutritional status using the MUST score, severity of dysphagia, anti-coagulation status and anatomical suitability. Each variable was allocated an appropriate categorical score. The maximum total score was 31 for the multivariate model and 40 for the RFH-GRS. Scores were then retrospectively allocated to the 234 gastrostomy referrals and the predictive validity for survival of the two scores compared.

Results The median (range) multivariate model score was 5 (1-11); the median RFH-GRS was 10 (2-18). The quartiles within these ranges were used to allocate patients to one of four score groups. Comparison of cumulative survival, based on these groups, showed that the RFH-GRS had superior predictive validity (Figure 1). Patients with a RFH-GRS of - have a low risk of an adverse outcome following gastrostomy placement; 6-10 a moderate risk, so they are likely to benefit; 1114 a medium to high risk so benefits may still outweigh the risk, and $\geq 15$ a high risk, making it unlikely that they would benefit from the procedure.

Conclusion The RFH-GRS is a simple, validated tool for the assessment of patients referred for gastrostomy placement. It utilises routinely collected data and hence can be easily applied.

\section{PWE-019 PROSPECTIVE VALIDATION OF THE ROYAL FREE HOSPITAL GASTROSTOMY RISK SCORE}

${ }^{1}$ Anastasia Russell-Dalton*, ${ }^{2}$ Catherine Fennessy, ${ }^{1}$ Marsha Y Morgan. ${ }^{1}$ UCL Institute for Liver and Digestive Health, Royal Free Campus, University College London, London, UK; ${ }^{2}$ Royal Free Hospital, Royal Free London NHS Foundation Trust, London, UK, UK

\subsection{6/gutjnl-2019-BSGAbstracts.350}

Introduction Percutaneous gastrostomy tubes are widely considered a safe and effective means for providing long-term enteral nutrition. However, placement is associated with high risk of death in the weeks following insertion. The Royal Free Hospital Gastrostomy Risk Score (RFH-GRS), was designed to facilitate risk-benefit assessment of patients referred for gastrostomy (Russell-Dalton et al. Clin Nutr 2019; 29:252.). It allocates a categorical score to eight factors viz. age, prognosis, nutritional status, severity of dysphagia, anatomical suitability, anti-coagulation status, comorbidities (Charlson Comorbidity Index) and recent infection; the maximum total is 40 . Scores are stratified to facilitate risk-benefit assessment viz. 0-5: low risk, likely benefit; 6-10: moderate risk, likely benefit; 11-14: medium-high risk, benefits may still outweigh the risk; and
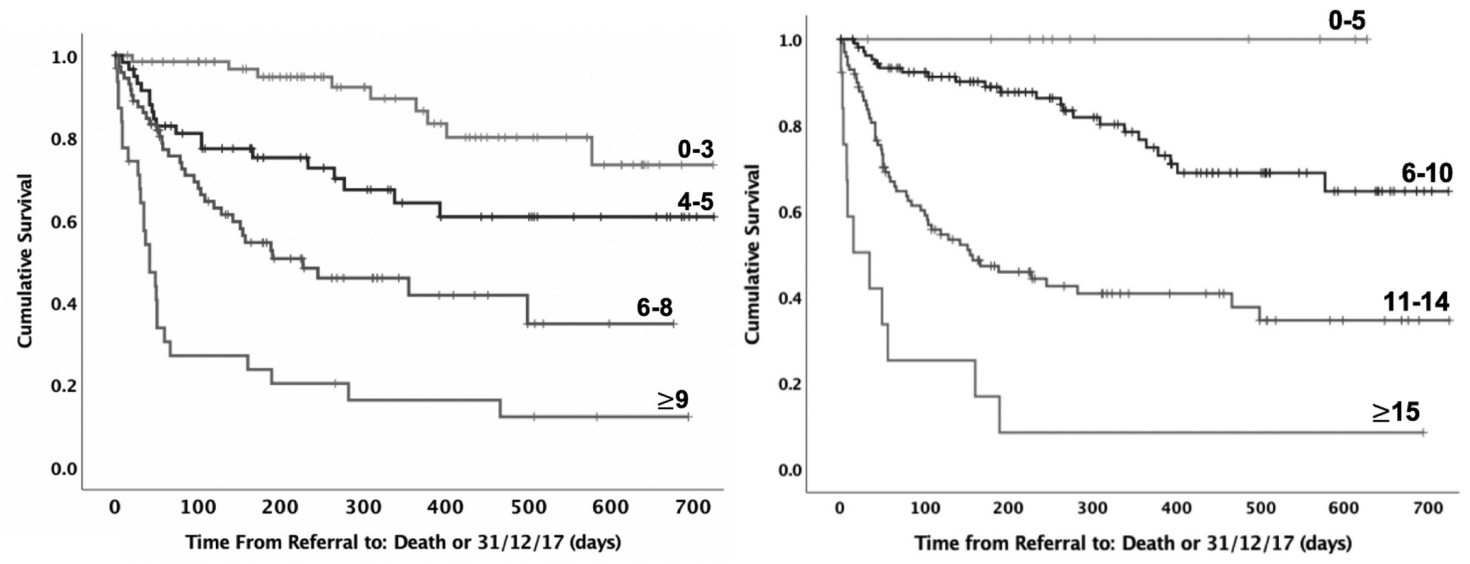

Abstract PWE-018 Figure 1 Cumulative survival in 234 gastrostomy candidates, by score 

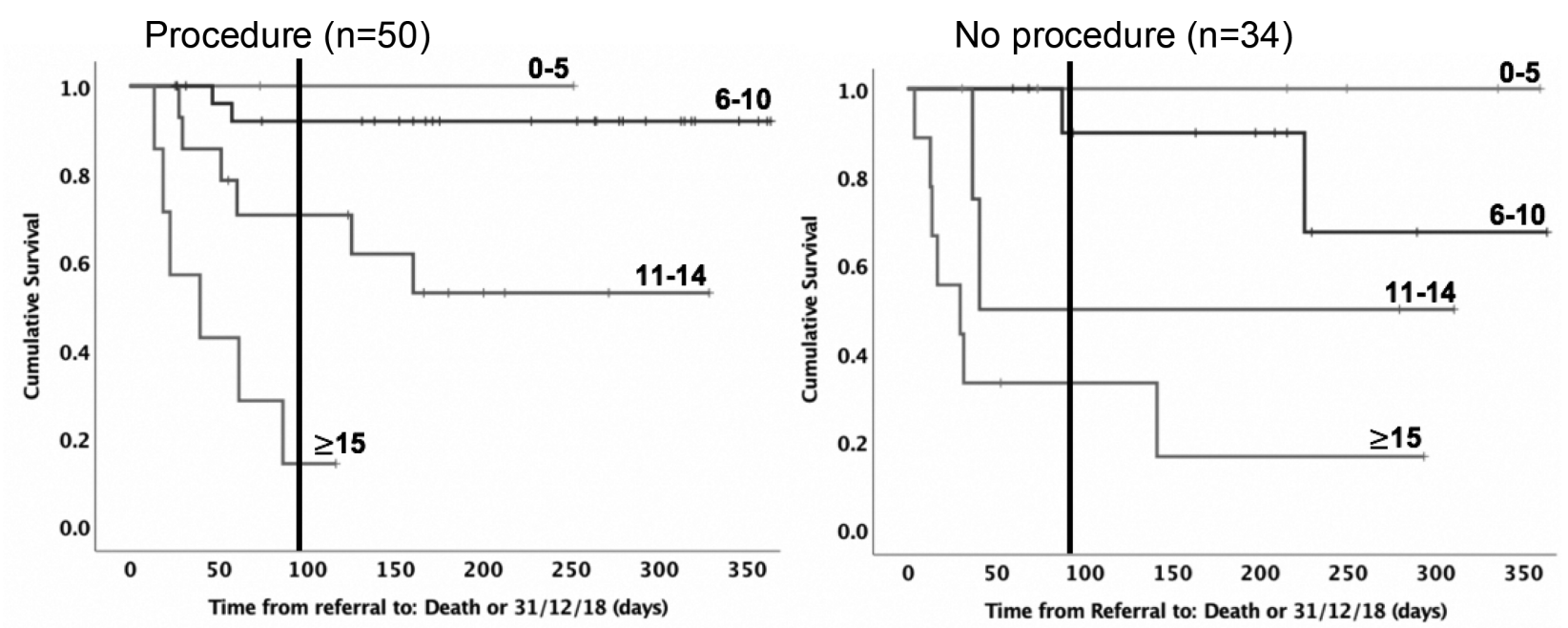

Abstract PWE-019 Figure 1 Cumulative survival in gastrostomy referrals, by procedural status

$\geq 15$ : high risk, unlikely to benefit. The aim of this one-year prospective study was to further evaluate the predictive validity of the RFH-GRS.

Methods All gastrostomy referrals from 1 January to 31 December 2018 were allocated a RFH-GRS score as part of their eligibility assessment. Patients were followed until death, or the end of the study period. Risk-benefit was assessed by comparing RFH-GRS and survival outcomes in patients who did and did not undergo the procedure.

Results Eighty-four patients were referred (58\% men; median [range] age, 72 [1-6] yr); dysphagia was the main indication in $71.4 \%$. Of the 84 referrals, 50 underwent placement, while 34 did not: too well $(n=11)$, too unwell $(n=14)$, declined $(n=3)$, unsuitable anatomy $(n=6)$. The median RFH-GRS was 10 (range -0 ). Patients with scores of -0 who underwent the procedure had a 93\% 90-day survival, compared with $89 \%$ in those who did not. However, 1-year survival rates were 93\% and $68 \%$ indicating clear long-term benefit (Figure 1). Patients with scores of 1-4 who underwent the procedure benefited in the short term with an $18 \%$ difference in 90-day survival (70\% vs $52 \%$ ) but 1 -year survival rates were similar at $55 \%$ and $50 \%$. Patients with scores $\geq 15$ who underwent the procedure had a significantly lower 90-day survival compared to those who did not (15\% vs 33\%).

Conclusion This prospective study provides further evidence to support the use of the RFH-GRS as a risk-benefit assessment tool in patients referred for gastrostomy placement.

\section{PWE-020 CATHETER-ASSOCIATED COMPLICATIONS INCLUDING DEEP VEIN THROMBOSIS IN A HPN COHORT: THE LEICESTER INTESTINAL FAILURE TEAM}

Syazeddy Samani*, Karuna Kodali, Melanie Baker, Dan Rogers, James Stewart. University Hospitals of Leicester NHS Trust, Leicester, UK

\subsection{6/gutjnl-2019-BSGAbstracts.351}

Introduction Deep vein thrombosis (DVT) is a recognized complication associated with central catheters and it is thought that peripherally inserted central venous catheters (PICC) were associated with higher rates compared to centrally inserted catheters (CVC) but studies performed were not always in patients with home parenteral nutrition (HPN). ${ }^{1,2}$ The aim of our analysis is to assess the incidence of catheter-associated deep vein thrombosis (CA-DVT) and evaluate different rates of catheter-associated complication (CA-C) between the PICC and CVC group in a HPN cohort.

Method This is a retrospective, single centre cohort analysis of patients on HPN who had either PICC or CVC. The number of catheters removed due to CA-C from the 1st of January 2018 to 31st of December 2018 was reviewed and expressed in episodes. Data on CA-C which consisted of CA-DVT, confirmed and suspected catheter-associated infection (CA-I), catheter-associated device dysfunction (CA-DD) including line fracture and occlusion were analysed. Rates were calculated based upon the number of catheter and catheter re-inserted during this time period. Cumulative episodes and rates were compared between the two groups. Fisher's exact test was used for statistical analysis.

Results 64.3\% ( $\mathrm{n}=45)$ had CVC (Hickman lines) and 35.7\% $(n=25)$ had PICC with 25 episodes of catheter removal $(n=19$ and $n=9$ respectively, multiple episodes per patient allowed). The overall rate of CA-DVT was low at $1.4 \%(n=1)$ and this was associated with a PICC. There was no reported deep vein thrombosis within the CVC group. The cumulative episodes of CA-C appeared higher in the CVC group compared to the PICC group although the difference in rates was not statistically significant $(42.2 \% \mathrm{n}=19$ vs $36 \% \mathrm{n}=9, \mathrm{p}=$ 0.7994). There were 9 episodes of CA-I and 10 episodes of CA-DD in the CVC group compared to 3 episodes of CA-I and 5 episodes of CA-DD in the PICC group. Statistical analysis showed no difference in rates of CA-I $(p=0.5029)$ or CADD $(p=1)$ between the two groups.

Conclusions This analysis showed an overall low rate of catheter-associated deep vein thrombosis within a HPN cohort in a 12 month period. There was no significant difference in rates of catheter-associated complication between PICC and CVC.

\section{REFERENCES}

1. Bonizzoli M, Batacchi $S$, et al. Peripherally inserted central venous catheters and central venous catheters related thrombosis in post-critical patients. Intensive Care Med. 2011;37(2.:284

2. Vineet Chopra et al. Risk of venous thromboembolism associated with peripherally inserted central catheters: A systematic review and meta-analysis. The Lancet. 2013;vol 382 issue 9889; 31-25. 